Bull. Austral. Math. Soc.

28B10, 03G12, 46L51, 81P10

VOL. $72(2005)$ [291-298]

\title{
BOUNDED VECTOR MEASURES ON EFFECT ALGEBRAS
}

\author{
Hong Taek Hwang, Longlu Li and Hunnam Kim
}

\begin{abstract}
Let $(L, \perp, \oplus, 0,1)$ be an effect algebra and $X$ a locally convex space with dual $X^{\prime}$. A function $\mu: L \rightarrow X$ is called a measure if $\mu(a \oplus b)=\mu(a)+\mu(b)$ whenever $a \perp b$ in $L$ and it is bounded if $\left\{\mu\left(a_{n}\right)\right\}_{n=1}^{\infty}$ is bounded for each orthogonal sequence $\left\{a_{n}\right\}$ in $L$. We establish five useful conditions that are equivalent to boundedness for vector measures on effect algebras.
\end{abstract}

\section{INTRODUCTION}

Following Gudder ([1]), quantum mechanics is a probabilistic theory, and a complete description of a quantum mechanical system is given by a probabilistic measure on its set events. This set of events fails to form a $\sigma$-field (or a $\sigma$-complete Boolean algebra) ([2]).

Motivated by the mathematical foundations of a quantum mechanics, so-called noncommutative measure theory has seen important developments. This theory replaces Boolean algebras by other structures (sometimes called quantum logics) such as orthoalgebras, effect algebras or $D$-posets. Note that effect algebra is a natural generalisation of Boolean algebras and orthoalgebras ([6]).

Recently, a series of basic principles of the usual measure theory, such as the BrooksJeweet theorem, Nikodym convergence theorem and Vitali-Hahn-Saks theorem, were also established for topological group valued measures defined on quantum logics $([2,6])$. However, the usual theory of locally convex space valued measures is quite plentiful so it is necessary to consider vector measure defined on quantum logics.

In this paper we establish boundedness results for locally convex space valued measures on effect algebras. For a usual vector measure, these boundedness results were established by Li Ronglu and Shin Min Kang ([4]) in 2000. Note that a recent result in locally convex space theory $([3,7])$ gives a new strong property of bounded vector measures (see Corollary 4 below).

Received 4th May, 2005

This paper was supported by Kumoh National Institute of Technology

Copyright Clearance Centre, Inc. Serial-fee code: 0004-9727/05 \$A2.00+0.00. 


\section{Preliminary}

Let $(L, \perp, \oplus, 0,1)$ be a given effect algebra (see [6]) and $X$ be a locally convex space with the dual $X^{\prime}$. A function $\mu: L \rightarrow X$ is a measure if $\mu(a \oplus b)=\mu(a)+\mu(b)$ whenever $a, b \in L$ and $a \perp b$. Let $a(L, X)$ be the family of $X$-valued measures on $L . \mu \in a(L, X)$ is $s$-bounded if $\mu\left(a_{n}\right) \rightarrow 0$ whenever $\left(a_{j}\right)_{j \in \mathbb{N}}$ is orthogonal sequence in $L$, and $s a(L, X)$ is the family of $s$-bounded measures. We say that $\mu \in a(L, X)$ is $\sigma$-additive if for every orthogonal sequence $\left(a_{n}\right)_{n \in \mathbb{N}}$ in $L$ such that $a=\oplus_{n \in \mathbb{N}} a_{n}$ exists in $L$, the series $\sum_{n=1}^{\infty} \mu\left(a_{n}\right)$ converges to $\mu(a)$, and $c a(L, X)$ is the family of $\sigma$-additive measures.

Each $\mu \in s a(L, X)$ is strongly additive, that is, for every orthogonal sequence $\left(a_{j}\right)_{j \in \mathrm{N}}$ in $L,\left\{\sum_{j=1}^{n} \mu\left(a_{j}\right)\right\}$ is Cauchy ([6, Corollary 3.4]) and, in general, both inclusions $c a(L, X) \subset s a(L, X)$ and $s a(L, X) \subset c a(L, X)$ are not true $([6,4])$.

Definition 1: $\quad \mu \in a(L, X)$ is said to be bounded if for every orthogonal sequence $\left(a_{j}\right)_{j \in \mathbf{N}}$ in $L$, the sequence $\left\{\mu\left(a_{j}\right)\right\}_{j=1}^{\infty}$ is bounded in X.

Let $b a(L, X)=\{\mu \in a(L, X): \mu$ is bounded $\}$.

Proposition 1. $s a(L, X) \subset b a(L, X)$.

This is an immediate consequence of the definition. We show that the converse is false.

Example. Let $L=\{A \subseteq \mathbb{N}: A$ is finite or $\mathbb{N} \backslash A$ is finite $\}$. For $A, B \in L$, we say that $A \perp B$ if $A \cap B=\emptyset$ and let $A \oplus B=A \cup B$ whenever $A \perp B$. Then $(L, \perp, \oplus, \emptyset, \mathbb{N})$ is an effect algebra. Define $\mu: L \rightarrow c_{0}$ by $\mu(A)=\chi_{A}$ if $A$ is finite and $-\chi_{\mathbb{N} \backslash A}$ if $\mathbb{N} \backslash A$ is finite, where $\chi_{B}$ is the characteristic function of $B \subseteq \mathbb{N}$. Then $\mu \in a\left(L, c_{0}\right)$. Since $\|\mu(A)\|_{\infty}=0$ or 1 for each $A \in L, \mu \in b a(L, X)$. However, $(\{n\})_{n \in \mathbb{N}}$ is orthogonal in $L$ but $\mu(\{n\})=\chi_{\{n\}}=e_{n}$ does not converge in $c_{0}$. This shows that $\mu \notin s a(L, X)$, $b a(L, X) \not \subset s a(L, X)$.

LEMMA 1. For $\mu \in a(L, X)$ the following are equivalent.

(i) $\mu \in b a(L, X)$.

(ii) For every orthogonal $A \subset L,\{\mu(a): a \in A\}$ is bounded.

(iii) For every orthogonal $A \subset L$ and $\mathcal{F}(\mathcal{A})$ the family of finite subsets of $A$, $\left\{\sum_{a \in F} \mu(a): F \in \mathcal{F}(\mathcal{A})\right\}$ is bounded.

Proof: (iii) $\Rightarrow$ (ii) $\Rightarrow$ (i) is obvious. Suppose that $\mu \in b a(L, X)$ but

$$
\left\{\sum_{a \in F} \mu(a): F \in \mathcal{F}(\mathcal{A})\right\}
$$

is not bounded. By the Mackey theorem $\left(\left[8\right.\right.$, p. 114]), there is an $f \in X^{\prime}$ such that

$$
\sup _{f \in \mathcal{F}(\mathcal{A})}\left|f\left(\sum_{a \in F} \mu(a)\right)\right|=+\infty .
$$


Then there is an $F_{1} \in \mathcal{F}(\mathcal{A})$ for which

$$
\left|f\left(\sum_{a \in F_{1}} \mu(a)\right)\right|>1
$$

and there is an $F_{2} \in \mathcal{F}(\mathcal{A})$ such that

$$
\left|f\left(\sum_{a \in F_{2}} \mu(a)\right)\right|>2+\sum_{a \in F_{1}}|f(\mu(a))| .
$$

Hence

$$
\left|f\left(\sum_{a \in F_{2} \backslash F_{1}} \mu(a)\right)\right|=\left|f\left(\sum_{a \in F_{2}} \mu(a)-\sum_{a \in F_{2} \cap F_{1}} \mu(a)\right)\right|>2 .
$$

There is an $F_{3} \in \mathcal{F}(\mathcal{A})$ such that

$$
\left|f\left(\sum_{a \in F_{3}} \mu(a)\right)\right|>3+\sum_{a \in F_{1} \cup F_{2}}|f(\mu(a))|
$$

and so

$$
\left|f\left(\sum_{a \in F_{3} \backslash\left(F_{1} \cup F_{2}\right)} \mu(a)\right)\right|=\left|f\left(\sum_{a \in F_{3}} \mu(a)-\sum_{a \in F_{3} \cap\left(F_{1} \cup F_{2}\right)} \mu(a)\right)\right|>3
$$

Continuing this construction inductively gives a sequence $\left\{F_{j}\right\} \subset \mathcal{F}(\mathcal{A})$ such that

$$
\left|f\left(\mu\left(\bigoplus_{a \in F_{n+1} \backslash\left(\bigcup_{j=1}^{n} F_{j}\right)} a\right)\right)\right|=\left|\sum_{a \in F_{n+1} \backslash\left(\bigcup_{j=1}^{n} F_{j}\right)} \mu(a)\right|>n+1
$$

$n=1,2,3, \ldots$.

Let $F_{1}^{\prime}=F_{1}, F_{n}{ }^{\prime}=F_{n}\left(\bigcup_{n=1}^{n-1} F_{j}\right)$ for $n \geqslant 2$. Then $\left\{F_{n}{ }^{\prime}\right\}_{n=1}^{\infty}$ is a pairwise disjoint sequence in $\mathcal{F}(\mathcal{A})$. In fact, if $n>m$ and $a \in F_{n}{ }^{\prime}$, then $a \in F_{n} \backslash\left(\bigcup_{j=1}^{n-1} F_{j}\right)$ so $a \notin F_{m}$ and $a \notin F_{m}{ }^{\prime}$. Since $A$ is orthogonal and $\left\{F_{n}{ }^{\prime}\right\}$ is a pairwise disjoint sequence of finite subset of $A$, it is easy to see that $\left(\bigoplus_{a \in F_{n}^{\prime}} a\right)_{n \in \mathbb{N}}$ is an orthogonal sequence in $L$ and so

$$
\left\{f\left(\mu\left(\bigoplus_{a \in F_{n+1} \backslash\left(\bigcup_{j=1}^{n} F_{j}\right)} a\right)\right)\right\}_{n=1}^{\infty}=\left\{f\left(\mu\left(\bigoplus_{a \in F_{n}^{\prime}} a\right)\right)\right\}_{n=1}^{\infty}
$$

is bounded because $\mu \in b a(L, X)$ and $f \in X^{\prime}$. This is a contradiction to (1) and, hence,

$$
\left\{\sum_{a \in F} \mu(a): F \in \mathcal{F}(\mathcal{A})\right\}
$$

is bounded. That is, (i) $\Rightarrow$ (iii) holds. 


\section{MAIN RESULTS}

Now we can state the main result as follows.

TheOREM 1. Let $(L, \perp, \oplus, 0,1)$ be a given effect algebra and $X$ be a locally convex space. Then for a (finitely additive) measure $\mu: L \rightarrow X$, that is $\mu \in a(L, X)$, the following conditions are equivalent.

1. $\mu \in b a(L, X)$. 2. For every orthogonal sequence $\left(a_{j}\right)_{j \in \mathbb{N}}$ in $L$ and $\left(t_{j}\right) \in c_{0},\left\{\sum_{j=1}^{n} t_{j} \mu\left(a_{j}\right)\right\}_{n=1}^{\infty}$
is a Cauchy sequence in $X$.

3. For every orthogonal sequence $\left(a_{j}\right)_{j \in \mathrm{N}}$ in $L$, the series $\left\{\sum_{j=1}^{n} \mu\left(a_{j}\right)\right\}_{n=1}^{\infty}$
is weakly conditionally Cauchy. That is,

$$
\sum_{j=1}^{n}\left|f\left(\mu\left(a_{j}\right)\right)\right|<+\infty, \forall f \in X^{\prime}
$$

4. For every orthogonal sequence $\left(a_{j}\right)_{j \in \mathbb{N}}$ in $L, \mu\left(a_{j}\right) \rightarrow 0$ weakly.

5. For every orthogonal $A \subset L$ and every increasing sequence $F_{1} \subseteq F_{2} \subseteq F_{3}$ $\subseteq \cdots$ of finite subsets of $A$,

$$
\frac{1}{k} \sum_{a \in F_{k}} t_{\alpha} \mu(a) \stackrel{\text { weak }}{\longrightarrow} 0 \text { for every choice of }\left|t_{\alpha}\right| \leqslant 1 \quad(a \in A)
$$

6. For every orthogonal $A \subset L$ and every increasing sequence $F_{1} \subseteq F_{2} \subseteq F_{3}$ $\subseteq \cdots$ of finite subsets of $A$,

$$
\lim _{k \rightarrow \infty} \frac{1}{k} \sum_{a \in F_{k}} t_{\alpha} \mu(a)=0 \text { for every choice of }\left|t_{\alpha}\right| \leqslant 1 \quad(\mathrm{a} \in \mathrm{A})
$$

ProOF: $(1) \Rightarrow(2)$. Let $\left(a_{j}\right)_{j \in \mathbb{N}}$ be orthogonal sequence in $L$ and

$$
B=\left\{\sum_{j \in \Delta} t_{j} \mu\left(a_{j}\right): \Delta \subset \mathbb{N} \text { is finite, } \sup _{j}\left|t_{j}\right| \leqslant 1\right\} .
$$

Let $f \in X^{\prime}$ and

$$
\begin{aligned}
& N_{1}=\left\{j \in \mathbb{N}: \operatorname{Re} f\left(\mu\left(a_{j}\right)\right) \geqslant 0\right\}, \text { and } \\
& N_{2}=\left\{j \in \mathbb{N}: \operatorname{Re} f\left(\mu\left(a_{j}\right)\right)<0\right\}, \\
& N_{3}=\left\{j \in \mathbb{N}: \operatorname{Im} f\left(\mu\left(a_{j}\right)\right) \geqslant 0\right\}, \\
& N_{4}=\left\{j \in \mathbb{N}: \operatorname{Im} f\left(\mu\left(a_{j}\right)\right)<0\right\} .
\end{aligned}
$$


Then for every finite $\Delta \subset \mathbb{N}$ and $\left|t_{j}\right| \leqslant 1, \forall j \in \mathbb{N}$,

$$
\begin{aligned}
\left|f\left(\sum_{j \in \Delta} t_{j} \mu\left(a_{j}\right)\right)\right| & \leqslant \sum_{j \in \Delta}\left|f\left(\mu\left(a_{j}\right)\right)\right| \\
& \leqslant \operatorname{Ref}\left(\sum_{j \in \Delta \cap N_{1}} \mu\left(a_{j}\right)\right)-\operatorname{Re} f\left(\sum_{j \in \Delta \cap N_{2}} \mu\left(a_{j}\right)\right) \\
& +\operatorname{Im} f\left(\sum_{j \in \Delta \cap N_{3}} \mu\left(a_{j}\right)\right)-\operatorname{Im} f\left(\sum_{j \in \Delta \cap N_{4}} \mu\left(a_{j}\right)\right) \\
& \leqslant\left|f\left(\sum_{j \in \Delta \cap N_{1}} \mu\left(a_{j}\right)\right)\right|+\left|f\left(\sum_{j \in \Delta \cap N_{2}} \mu\left(a_{j}\right)\right)\right| \\
& +\left|f\left(\sum_{j \in \Delta \cap N_{3}} \mu\left(a_{j}\right)\right)\right|+\left|f\left(\sum_{j \in \Delta \cap N_{4}} \mu\left(a_{j}\right)\right)\right| \\
& \leqslant 4 \sup \left\{\left|f\left(\sum_{j \in F} \mu\left(a_{j}\right)\right)\right|: F \subset \mathbb{N} \text { is finite }\right\} \\
& =4 M_{f} .
\end{aligned}
$$

Since $\left(a_{j}\right)_{j \in \mathbb{N}}$ is orthogonal in $L$ and $\mu \in b a(L, X)$, it follows from Lemma 2 that $\left\{\sum_{j \in F} \mu\left(a_{j}\right): F \subset \mathbb{N}\right.$ is finite $\}$ is bounded and so $M_{f}<+\infty$ for each $f \in X^{\prime}$. This shows that $B$ is weakly bounded and hence, $B$ is bounded by the Mackey theorem.

Now let $\left(t_{j}\right) \in c_{0}$ and $\delta_{k}=\sup _{j \geqslant k}\left|t_{j}\right|, \forall k \in \mathbb{N}$. Then $\delta_{k} \rightarrow 0$. Without loss of generality, we assume that $t_{j} \neq 0$ for infinitely many $j$. Then $\delta_{k}>0$ for all $k \in \mathbb{N}$.

Let $U \in \mathcal{N}(\mathcal{X})$, the family of neighbourhoods of $0 \in X$. Since $B$ is bounded, there is an $\varepsilon>0$ such that

$$
\alpha \sum_{j \in \Delta} s_{j} \mu\left(a_{j}\right) \in U, \quad \forall|\alpha| \leqslant \varepsilon, \quad \sum_{j \in \Delta} s_{j} \mu\left(a_{j}\right) \in B
$$

Then there is a $k_{0} \in \mathbb{N}$ for which $0<\delta_{k}<\varepsilon, \forall k>k_{0}$, and

$$
\sum_{j=k}^{m} t_{j} \mu\left(a_{j}\right)=\delta_{k} \sum_{j=k}^{m}\left(t_{j} / \delta_{k}\right) \mu\left(a_{j}\right) \in U, \forall m \geqslant k>k_{0},
$$

that is, $\left\{\sum_{j=1}^{n} t_{j} \mu\left(a_{j}\right)\right\}_{n=1}^{\infty}$ is Cauchy.

$(2) \Rightarrow(3)$ : Let $\left(a_{j}\right)_{j \in \mathbf{N}}$ be orthogonal in $L$ and $f \in X^{\prime}$. By (2), $\sum_{j=1}^{\infty} t_{j} f\left(\mu\left(a_{j}\right)\right)$ converges for each $\left(t_{j}\right) \in c_{0}$ and, hence, $\sum_{j=1}^{\infty}\left|f\left(\mu\left(a_{j}\right)\right)\right|<+\infty$.

(3) $\Rightarrow(4)$ : Obvious.

(4) $\Rightarrow(1)$ : For every orthogonal $\left(a_{j}\right)_{j \in \mathbb{N}} \subset L, \mu\left(a_{j}\right) \stackrel{\text { weak }}{\longrightarrow} 0$ so $\left\{\mu\left(a_{j}\right)\right\}_{j=1}^{\infty}$ is weakly bounded and, hence, $\left\{\mu\left(a_{j}\right)\right\}_{j=1}^{\infty}$ is bounded by the Mackey theorem. 
(1) $\Rightarrow(6)$ : Let $A \subset L$ be orthogonal. It is similar to the proof of the implication (1) $\Rightarrow(2)$.

Lemma 2 implies that

$$
B=\left\{\sum_{a \in F} t_{a} \mu(a): F \subset A \text { is finite, }\left|t_{a}\right| \leqslant 1(a \in A)\right\}
$$

is bounded for $\mu \in b a(L, X)$. Hence, (1) implies (6).

(6) $\Rightarrow(5)$ : Obvious.

(5) $\Rightarrow(1)$ : Suppose that $\left(a_{j}\right)_{j \in \mathbb{N}}$ is orthogonal sequence in $L$ but $\mu\left(a_{j}\right) \stackrel{\text { weak }}{\longleftrightarrow} 0$. Then there exist $\varepsilon>0, f \in X^{\prime}$ and $j_{1}<j_{2}<j_{3}<\cdots$ in $\mathbb{N}$ such that

$$
\left|f\left(\mu\left(a_{j_{\nu}}\right)\right)\right|>\varepsilon, \nu=1,2,3, \ldots \text {. }
$$

Let

$$
F_{1}=\left\{a_{j_{1}}\right\}, \quad F_{2}=\left\{a_{j_{1}}, a_{j_{2}}\right\}, \quad F_{3}=\left\{a_{j_{1}}, a_{j_{2}}, a_{j_{3}}\right\}, \ldots,
$$

and pick a sequence $\left\{t_{\nu} \subset \mathbb{C}\right\}$ for which $\left|t_{\nu}\right|=1$ and

$$
t_{\nu} f\left(\mu\left(a_{j_{\nu}}\right)\right)=\left|f\left(\mu\left(a_{j_{\nu}}\right)\right)\right|, \quad \nu=1,2,3, \ldots
$$

Then

$$
\begin{aligned}
f\left(\frac{1}{k} \sum_{a_{j_{\nu}} \in F_{k}} t_{\nu} \mu\left(a_{j_{\nu}}\right)\right) & =\frac{1}{k} \sum_{a_{j_{\nu}} \in F_{k}} t_{\nu} f\left(\mu\left(a_{j_{\nu}}\right)\right) \\
& =\frac{1}{k} \sum_{\nu=1}^{k}\left|f\left(\mu\left(a_{j_{\nu}}\right)\right)\right| \geqslant \varepsilon \text { for all } k .
\end{aligned}
$$

This contradicts (5) and so $\mu\left(a_{j}\right) \rightarrow 0$ weakly. Thus $(5) \Rightarrow(4) \Rightarrow(1)$ holds.

For a locally convex space $X$ and its dual $X^{\prime}$, the strongest admissible topology $\beta\left(X, X^{\prime}\right)$ is just the topology of uniform convergence on each weak * bounded subset of $X^{\prime}$.

COROLlary 1. Let $(L, \perp, \oplus, 0,1)$ be an effect algebra and $X$ a sequentially complete locally convex space. Then a measure $\mu: L \rightarrow X$ is bounded (that is, $\mu \in b a(L, X)$ ) if and only if for every orthogonal $\left(a_{j}\right)_{j \in \mathbf{N}} \subset L$ and $\left(t_{j}\right) \in c_{0}$, the series $\sum_{j=1}^{\infty} t_{j} \mu\left(a_{j}\right)$ is $\beta\left(X, X^{\prime}\right)$-convergent.

PROOF: Let $\left(a_{j}\right)_{j \in \mathbf{N}}$ be orthogonal sequence in $L$. By Theorem $1, \sum_{j=1}^{\infty} t_{j} \mu\left(a_{j}\right)$ converges for each $\left(t_{j}\right) \in c_{0}$ since $X$ is sequentially complete. Since the $c_{0}$-multiplier convergence is an invariant on all admissible topologies $([3,7]), \sum_{j=1}^{\infty} t_{j} \mu\left(a_{j}\right)$ is $\beta\left(X, X^{\prime}\right)$ -
convergent. For each $\left(t_{j}\right) \in c_{0}$. 
Reflexive locally convex spaces are weakly sequentially complete ([8, p. 153]), and every weakly sequentially complete locally convex space contains no copy of $\left(c_{0},\|\|_{\infty}\right)$ ([5, Theorem 5]). If a locally convex space $X$ contains no copy of $\left(c_{0},\|\|_{\infty}\right)$, then $X$ has a series of nice properties, for example, every continuous linear operator $T: c_{0} \rightarrow X$ is both compact and sequentially compact. That is, for every bounded $B \subset c_{0}, \overline{T(B)}$ is both compact and sequentially compact ([5, Theorem 4]).

THEOREM 2. For a sequentially complete locally convex space $X$, the following conditions are equivalent.

(a) $X$ contains no copy of $\left(c_{0},\|\cdot\|_{\infty}\right)$,

(b) For every effect algebra $L$ and $\mu \in b a(L, X), \sum_{j=1}^{\infty} t_{j} \mu\left(a_{j}\right)$ converges whenever $\left(a_{j}\right)_{j \in \mathbf{N}}$ is orthogonal sequence in $L$ and $\left(t_{j}\right) \in l^{\infty}$,

(c) For every effect algebra $L, b a(L, X)=s a(L, X)$.

PRoOF: $(\mathrm{a}) \Rightarrow$ (b): Let $L$ be an effect algebra and $\left(a_{j}\right)_{j \in \mathbf{N}}$ an orthogonal sequence in $L$. By Corollary $1, \mu \in b a(L, X)$ implies that $\sum_{j=1}^{\infty} t_{j} \mu\left(a_{j}\right)$ converges for each $\left(t_{j}\right) \in c_{0}$. But, since $X$ contains no copy of $\left(c_{0},\|\cdot\|_{\infty}\right)$, it follows from [5, Theorem 1$]$ that $\sum_{j=1}^{\infty} t_{j} \mu\left(a_{j}\right)$ converges for each $\left(t_{j}\right) \in l^{\infty}$.

(b) $\Rightarrow$ (c): Trivial.

(c) $\Rightarrow$ (a): Example 1 .

\section{REFERENCES}

[1] S.P. Gudder, Quantum probability (Academic Press, Boston, 1989).

[2] E.D. Habil, 'Brooks-Jewett and Nikodym convergence theorems for orthoalgebras that have the weak subsequential interpolation property', Internat. J. Theoret. Phys. 34 (1994), 465-491.

[3] R.L. Li, R.C. Cui and M.H. Zhao, 'Invariants on all admissible polar topologies', Chinese Ann. Math. Ser. A 19 (1998), 289-294.

[4] R. Li and S.M. Kang, 'Characterizations of bounded vector measures', Bull. Korean Math. Soc. 37 (2000), 209-215.

[5] R.L. Li and Q.Y. Bu, 'Locally convex spaces containing no copy of $c_{0}$ ', J. Math. Anal. Appl. 172 (1993), 205-211.

[6] F.G Mazario, 'Convergence theorems for topological group valued measures en effect algebras', Bull. Austral. Math. Soc. 64 (2001), 213-231.

[7] C. Swartz and C. Stuart, 'Orlicz-Pettis theorems for multiplier convergent series', $Z$. Anal. Anwendungen 17 (1998), 805-811.

[8] A. Wilansky, Modern methods in topological vector spaces (McGraw-Hill, New York, 1978). 
Department of Applied Mathematics Kumoh National Institute of Technology Gumi, Gyeongbuk 730701

South Korea

email: mathvision@hotmail.com

Department of Applied Mathematics Kumob National Institute of Technology Gyeongbuk Foreign Language High School South Korea
Department of Mathematics Harbin Institute of Technology China 